\title{
Interference competition between an invasive parakeet and native bird species at feeding sites
}

\author{
Marine Le Louarn ${ }^{1,2}$ (1) $\cdot$ Bertrand Couillens ${ }^{2} \cdot$ Magali Deschamps-Cottin $^{1}$ • \\ Philippe Clergeau ${ }^{2}$
}

Received: 22 July 2015/Accepted: 8 June 2016/Published online: 16 June 2016

(C) Japan Ethological Society and Springer Japan 2016

\begin{abstract}
Interference competition has proved to be a factor of successful establishment of invasive species. This type of competition may have a stronger impact when native species have temporal niche overlap with the invasive species. The ring-necked parakeet Psittacula krameri has been successfully introduced in many countries and its interspecific agonistic behavior has already been reported. The purpose of this study is to analyze the territorial and preemptive interference competition between the ringnecked parakeet and native bird species in a recently colonized area. We used an empirical approach by recording video sequences in gardens equipped with bird feeders in winter. Our results showed that the ring-necked parakeet was the most frequent species at the feeders. Several native species showed temporal niche overlap with the ringnecked parakeet, the highest overlap being with the starling Sturnus vulgaris. The starling was also the species most impacted by interference competition with the parakeet. Our study suggests that, by being most frequently present at the feeders, by demonstrating the most agonistic behavior and by hindering access to food of the other species, the ring-necked parakeet is a superior competitor and may compete with native bird species.
\end{abstract}

Electronic supplementary material The online version of this article (doi:10.1007/s10164-016-0474-8) contains supplementary material, which is available to authorized users.

Marine Le Louarn

marine.le-louarn@univ-amu.fr

1 IRD, LPED UMR_D 151, Aix Marseille Univ, 13331 Marseille, France

2 UMR CESCO, Muséum National d'Histoire Naturelle, 55 rue Buffon, CP51, 75005 Paris, France
Keywords Agonistic behavior - Psittacula krameri . Feeder · Introduced species - Temporal niche overlap

\section{Introduction}

Several mechanisms can play a role in the success of establishment of introduced bird species: for example high propagule pressure (Cassey et al. 2004), low brood value (Sol et al. 2012), monochromatism, sedentarism, large body size (Blackburn et al. 2009) and some behavioral traits. Species with behavioral flexibility, linked to a larger brain, have shown a higher probability of successful establishment (Sol et al. 2002). Among these traits, agonistic behaviors [sensu Johnson (1972)-fighting between animals and competitive behavior that includes threats, offensive attacks and defensive fighting] may allow primary access to scarce resources (Wilson 1975) and induce interference competition. Interference competition encompasses processes by which the behaviors of one species prevent the access to resources by other species (Maurer 1984) and can be divided into two main types (Schoener 1983): territorial competition (when a species hinders the access to resources of other species by agonistic behavior) and preemptive competition (when a species, simply by its presence, gains full access to resources by controlling space). Interference competition has proved to be a process enabling invasive species to be competitively superior to native species through more efficient utilization of resources [e.g., in ants (Human and Gordon 1996; Lach 2005); wasps (Grangier and Lester 2011); reptiles (Case et al. 1994); plants (Huenneke and Thomson 2003)]. The intensity of interspecific competition may be increased by overlap in temporal activity pattern among species that share resources (Pianka 1973). 
Among avian invasive species, the ring-necked parakeet (or rose-ringed parakeet) Psittacula krameri, is one of the most widely introduced in the world. This bird originates from India and Africa and has established populations in at least 35 countries (Butler 2003). Its superior competitiveness versus native nuthatches Sitta europaea for cavity access in Belgium has been experimentally shown (Strubbe et al. 2010; Strubbe and Matthysen 2009) as well as its impacts on the foraging behavior of native birds in UK (Peck et al. 2014). Several authors have reported agonistic behaviors of the ring-necked parakeet next to their nest sites: lethal attacks on a Leisler's bat (Nyctalus leisleri) in Italy (Menchetti et al. 2014), on black rats (Rattus rattus) in Spain (Hernández-Brito et al. 2014b) and on several competitor and predator species in Spain (Hernández-Brito et al. 2014a). In the Paris area in France, a lethal attack by a ring-necked parakeet on an adult red squirrel (Sciurus vulgaris) has been reported (Clergeau et al. 2009). In the Paris region, populations of the ring-necked parakeet are increasing exponentially each year (Clergeau et al. 2015) and it has been suggested that one of the drivers of its demographic success may be its ability to efficiently exploit supplementary food provided by humans (Clergeau and Vergnes 2011).

Here, we have examined the interference competition and the temporal niche overlap between the ring-necked parakeet and other species with regard to supplementary food resources in winter. We built an observation protocol using gardens equipped with bird feeders and cameras in the Paris area in France. Considering the large use of anthropogenic food by the ring-necked parakeet and its agonistic behavior at breeding sites, we aim to explore its foraging behavior and the response of native bird species to this new invader. More specifically, we examined two types of interference competition, territorial and preemptive, and tested their links with the temporal niche overlap between the ring-necked parakeet and native bird species present at the feeders.

\section{Materials and methods}

\section{Experimental protocol}

The study was conducted during the harsh winter period that concentrated birds at feeding sites. We chose to use gardens with existing bird feeders in order to avoid the problem of the time required by birds to find a new resource and become accustomed to the location.

After a request made to local ornithological associations, eight private gardens were selected on the basis of regular filling of feeders with seeds and the ease of access of the feeders. All the gardens were situated in residential areas of the southern Paris suburbs, Ile-de-France region: six in the city of Antony [the sector most densely populated by the ring-necked parakeet in the region (Clergeau et al. 2015)] and two in the city of Sucy-en-Brie (a more recently colonized sector with fewer parakeets). The parakeet population has been settled since the 1980s and has increased in particular in these sectors to around 5000 individuals (Clergeau et al. 2015). The selected gardens presented similar bird feeders attached to branches at a height of 1-2 $\mathrm{m}$ above the ground that were installed several years ago. During the entire study period, feeders were supplied with a mix of seeds composed mainly of sunflower. To reduce the risk of pseudo-replication with the same individuals at different feeders, the gardens were at least $500 \mathrm{~m}$ apart.

Within each garden, we used a camera (Naturacam MP) placed $3 \mathrm{~m}$ from the feeder to record an area of about $3 \mathrm{~m}$ around the feeder, thus including possible perching sites around the feeders. The cameras were active during the morning, when foraging activity of birds is generally high (McNamara et al. 1994), between 07:45 a.m. and 12:00 p.m. from 15 November 2013 to 15 February 2014. For each movement within the camera's range, a 30-s video sequence was recorded with a 10-min break between each sequence. This interval made it possible to obtain independent data sequences.

\section{Data analysis}

For each video sequence, we characterized the structural aspects of the avian assemblage by noting the following parameters: presence of species, maximum number of feeding individuals (present on the bird feeder) per species, and maximum number of non-feeding individuals (present next to the bird feeder) per species. For ecological and statistical reasons, we have restricted the analyses to the most abundant species [those species with a relative frequency of occurrence in the video sequences greater than the inverse of total species richness (Camargo 1993)].

To determine the effect of body mass in the interference competition between the ring-necked parakeet and the native bird species, we grouped the most abundant species into three guilds: small species $(0-50 \mathrm{~g})$, medium species $(50-100 \mathrm{~g})$ and large species $(>100 \mathrm{~g})$. The average body mass for each species was based on Dunning (2007).

For the purpose of analyzing the potential temporal niche overlap between the ring-necked parakeet and the other bird species, we recorded the time of feeding activity for each species during each video sequence. We then combined the video sequences in 15-min sequences. Number of feeding events for each species during each time interval represented an estimate of resource use. Pairwise interspecific differences in times of feeding 
activity were evaluated with Kolmogorov-Smirnov twosample tests (Siegel 1956) using R (R Core Development Team 2015). Temporal niche overlap was measured with the Czekanowski index (Feinsinger et al. 1981). This symmetrical index ranges from zero (for two species with completely different temporal activity patterns) to one (for two species that have identical temporal activity patterns). The overlap analyses were performed in the TimeOverlap program (Castro-Arellano et al. 2010) using the randomization algorithm Rosario. This algorithm generates a null distribution of overlap index values. For each pair of species, randomization was iterated 10,000 times and significance was calculated by comparing each empirical index with the associated null distribution. In order to avoid the omission of relevant biological results, and based on Roback and Askins (2005), which states that corrections for multiple tests are not recommended for exploratory analyses, we did not apply $p$-value correction to the pairwise comparisons of temporal overlap between the ringnecked parakeet and the native birds species.

In order to determine whether invasive parakeets were able to exclude native birds from food resources by a specific behavior in our gardens, we analyzed the territorial interference competition events (TICE) of all the bird species. When TICE occurred, the following terminology was used:

- If species $x$ was feeding and species $y$ displaced species $x$ from the feeder, species $x$ was considered the "loser" and species $y$ the "winner". We only considered the aggressive displacements caused by threat displays (open beaks and/or wings, and/or bristled feathers) and direct attacks (diving flight, and/or physical contact).

- If species $x$ was feeding and species $y$ did not displace species $x$ from the feeder, despite aggressive behavior, species $x$ was considered the winner and species $y$ the loser.

- If two species were feeding at a feeder and one of them displaced the other, we considered that there was a TICE only if there was aggressive behavior (see above).

- The species that initiated the TICE was considered the "initiator" and the other species involved the "victim".

For each TICE, the number of individuals of each species involved was noted.

Due to their relatively small size, bird feeders can only offer access to a small number of individuals. Some large and gregarious species, such as the ring-necked parakeet, may passively exclude smaller species by occupying all the available space on the feeders. We called preemptive interference competition events (PICE) those where one species was on a feeder and another species perched next to the feeder. For each PICE, we noted the duration of the event, the species involved and the number of individuals per species.

Due to the gregarious behavior of the ring-necked parakeet, we tested the potential effect of the number of parakeets involved in PICE and TICE by using a general linear model (GLM), with a binomial error distribution and a logistic link function using $\mathrm{R}$ ( $\mathrm{R}$ Core Development Team 2015).

\section{Results}

The cameras filmed 1198 video sequences. We only considered the video sequences with at least one feeding bird for further analyses. In total, 533 video sequences showing feeding birds $(44.5 \%, n=1198)$ were recorded, which represents more than $4 \mathrm{~h}$ of video sequences, analyzed by a single observer.

\section{Structural aspects of the bird assemblage}

Nineteen bird species were recorded feeding at bird feeders: the ring-necked parakeet (Psittacula krameri), the great tit (Parus major), the Eurasian blue tit (Cyanistes caeruleus), the coal tit (Periparus ater), the Eurasian collared-dove (Streptopelia decaocto), the house sparrow (Passer domesticus), the European greenfinch (Chloris chloris), the rock dove (Columba livia), the common chaffinch (Fringilla coelebs), the European robin (Erithacus rubecula), the common starling (Sturnus vulgaris), the Eurasian tree sparrow (Passer montanus), the Eurasian magpie (Pica pica), the Eurasian bullfinch (Pyrrhula pyrrhula), the long-tailed tit (Aegithalos caudatus), the common blackbird (Turdus merula), the Eurasian jay (Garrulus glandarius), the brambling (Fringilla montifringilla) and the European green woodpecker (Picus viridis). After deletion of rare species, we conducted the analysis on eight bird species: five small species, one medium species and two large species (Table 1).

The ring-necked parakeet was the most frequently present species in the video sequences $(43.5 \%, n=533$, binomial test, $p<0.01)$, followed by the great tit $(27.6 \%$, $n=533$ ). The other species were less present, with a maximum of $16.3 \%$ for the Eurasian blue tit and a minimum of $7.3 \%$ for the common chaffinch (Table 1). The starling and the ring-necked parakeet were the most numerous, with respectively 2.31 and 1.90 individuals on average at the same time at the feeders (Fig. 1). Starlings were recorded with more than one individual in $81.5 \%$ of all sequences and the ring-necked parakeet in $74.8 \%$, which represent the highest percentages, all species included. 
Table 1 The most abundant bird species recorded in the video sequences

\begin{tabular}{llll}
\hline Species & & $\begin{array}{l}\text { Frequency of presence in the } \\
\text { video sequences }(n=533)(\%)\end{array}$ & Body mass guild \\
\hline Psittacula krameri & Ring-necked parakeet & 43.5 & Large \\
Parus major & Great tit & 27.6 & Small \\
Cyanistes caeruleus & Eurasian blue tit & 16.3 & Small \\
Streptopelia decaocto & Eurasian collared dove & 14.8 & Large \\
Passer domesticus & House sparrow & 10.7 & Small \\
Fringilla coelebs & Common chaffinch & 7.3 & Small \\
Erithacus rubecula & European robin & 8.8 & Small \\
Sturnus vulgaris & Common starling & 11.4 & Medium
\end{tabular}

Guilds were formed on the basis of average body mass (Dunning 2007): $0-50 \mathrm{~g}=$ small species, 50-100 $\mathrm{g}=$ medium species and $>100 \mathrm{~g}=$ large species

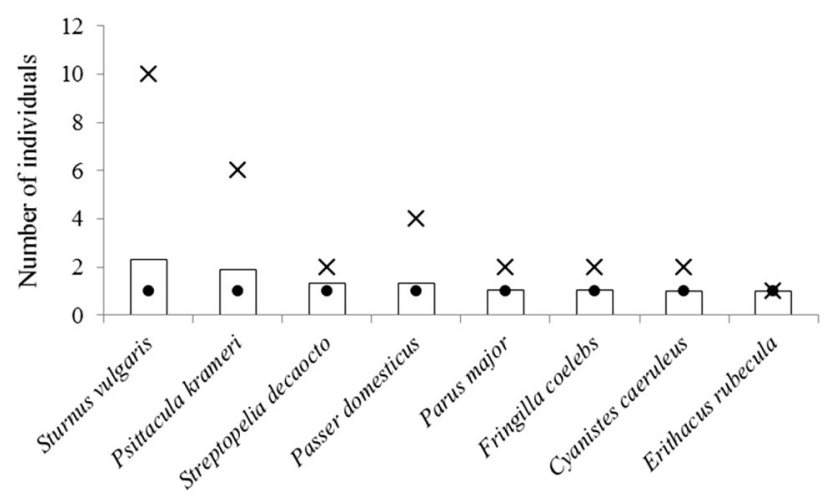

Fig. 1 Number of feeding birds at the feeders. Black crosses represent the maximum number of individuals per species feeding at the same time at the feeders, black circles the minimum, and white bars the average $(n=533)$

The average species richness over the full set of video sequences was 1.50 species. When the parakeet was absent, the number of feeding birds of other species was significantly higher than when the parakeet was present in the video (Mann-Whitney rank sum test, $p<0.05$ ). In contrast, when the parakeet was absent, the number of nonfeeding birds of other species was significantly lower than when the parakeet was present in the video (Mann-Whitney rank sum test, $p<0.001$ ).

\section{Species' temporal activity patterns at the feeders}

Abundant species showed a heterogeneous use of time during the morning and were present at different times at the feeders (Kruskal-Wallis test, $\chi^{2}=27.04, d f=7$, $p<0.001$, Supplementary Fig. S1). Some species showed temporal activity peaks whereas other showed a more multimodal time use. The ring-necked parakeet began arriving at 08:45 a.m. and its feeding activity peak was around 09:45 a.m. The European robin was present earlier than the other species, with an activity peak around 08:15 a.m. The duration of feeding activity was different between the bird species (Kruskal-Wallis test, $\chi^{2}=50.35, d f=7$, $p<0.001$ ). The tits spent less time feeding (median $<5 \mathrm{~s}$ ) than the ring-necked parakeet and the Eurasian collared dove (median $30 \mathrm{~s}$, i.e., the whole duration of the video sequence).

The Czekanowski pairwise overlap indexes were significantly different than expected by chance for three of seven comparisons between the parakeet and the other species (Supplementary Table S2) The highest temporal overlap of the ring-necked parakeet was with the starling (Czekanowski index $=0.63, p=0.04$ ), and the European robin had the least temporal overlap with the ring-necked parakeet (Czekanowski index $=0.38, p=0.02$; Fig. 2).

\section{Territorial interference competition event}

Sixty-five TICE were recorded involving six species (the eight most abundant species with the exception of the European robin and the common chaffinch). The species most frequently involved in a TICE was the ring-necked parakeet with 33 TICE, representing more than half of all the events $(50.8 \%, n=65$, binomial test, $p<0.01)$. This species was the initiator in $60.6 \%$ of the TICE in which it was involved and was the winner in $78.8 \%$ of the cases. The starling was the species most frequently involved in the TICE with the ring-necked parakeet $(39.4 \%$ of all the parakeet's TICE, $n=33$, binomial test, $p<0.05$ ) and the parakeet interacted significantly more with the starling than with the other bird species (binomial test, $p<0.05$ ).

Considering all the TICE between the ring-necked parakeet and another species, the parakeet initiated and won the interactions in more than $60 \%$ of all cases, with the exception of the great tit which initiated the TICE in more than half of all cases $(57.1 \%, n=7)$.

There is no significant difference in the TICE with the ring-necked parakeet between the different guilds of size. 
Fig. 2 Example of daily temporal feeding activity comparisons between Psittacula krameri and two other species: a no temporal overlap with European robin, b temporal overlap with starling. Data were collected between 07:45 a.m. and 12:00 p.m. Each circle inside the radar charts represents $5 \%$ of the feeding bird presence in the full set of video sequences $(n=533)$
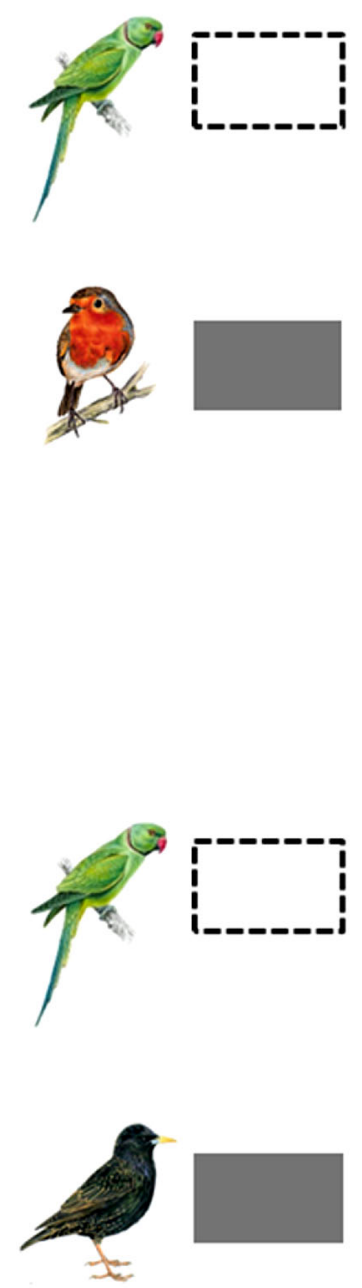

(a) Daily temporal activity Psittacula krameri - Erithacus rubecula Czekanowski index $=\mathbf{0 . 3 8}$

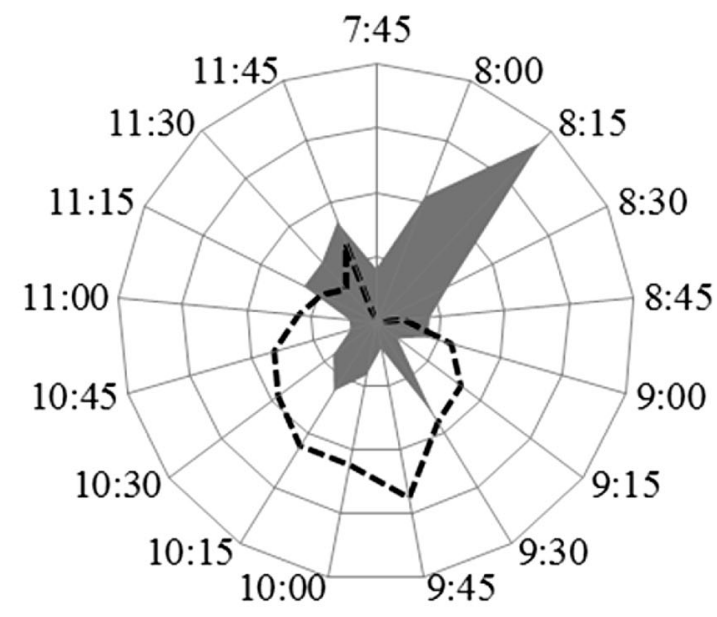

(b) Daily temporal activity Psittacula krameri - Sturnus vulgaris Czekanowski index $=\mathbf{0 . 6 3}$

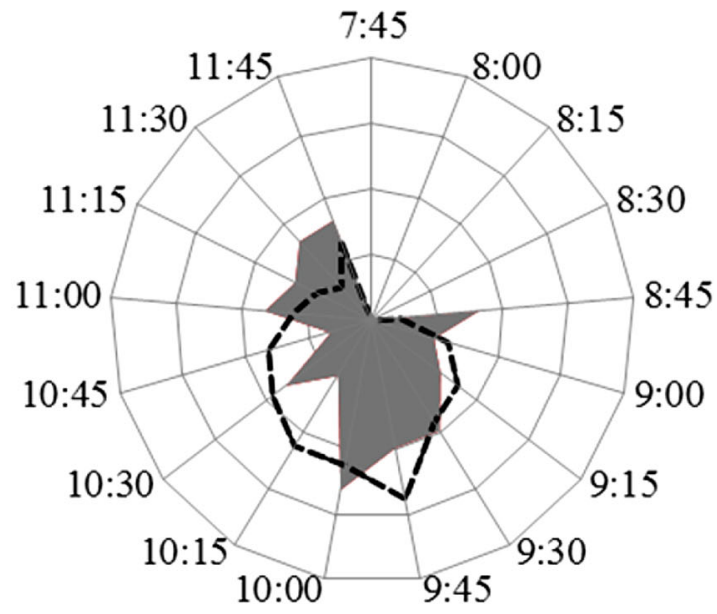

necked parakeet $(40.8 \%, n=233$, binomial test, $p<0.01)$ and the average duration of the PICE caused by the ring-necked parakeet was significantly longer than the PICE caused by other species (Mann-Whitney rank sum test, $p<0.001)$. The PICE caused by the parakeet involved common starlings $(34.7 \%, n=95)$, Eurasian collared doves $(20.4 \%, n=95)$, great tits $(18.4 \%, n=95)$, blue tits $(12.2 \%, n=95)$, common chaffinches $(10.2 \%$, $n=95)$ and European robins $(4.1 \%, n=95)$. We found no significant effect of the number of parakeets present on the feeder at the moment of the event on the number of PICE caused by this species (GLM, $\chi^{2}=3.48, n=95$, $p>0.05$ ). By analyzing the number of PICE caused by the ring-necked parakeet on the different body mass guilds (Table 1), we found that the probability that a species was involved in a PICE caused by the ring-necked parakeet 
increased with a decrease in body mass (GLM, $\chi^{2}=2.54$, $p<0.05)$.

\section{Discussion}

Behavioral traits play a key role in the post-establishment phase (Chapple et al. 2012). The ring-necked parakeet has successfully established viable populations in many countries, and several authors have reported its interspecific agonistic behavior. Our study shows the territorial and preemptive interference competition for supplementary food resources between the ring-necked parakeet and native bird species. This competition is induced by the agonistic behavior of this invasive parakeet (territorial interference) and also by its passive monopolization of the food resources (preemptive interference) at the feeders.

Our results showed that the ring-necked parakeet was the most aggressive species at the feeders. This species initiated and won the aggressive interactions in most of the cases. These results are congruent with the findings of Peck et al. (2014) and support the hypothesis that the ringnecked parakeet may outcompete native bird species by having an effect on their foraging behavior. Its agonistic behavior may induce competitive advantages such as access to greater amounts of food and may indirectly cause a lower fitness and individual physical condition for the native species. In our study, the frequency of interference competition was not parakeet group-size dependent. But the lighter the species, the more "bullying" the ring-necked parakeet was. The effect of body size in interspecific interference competition has been well documented, and the superior competitiveness of one species towards another leads to asymmetrical competition (Schoener 1983). According to theory, the smaller species should develop behaviors that lead to the avoidance of conflict and the reduction of aggressions by larger species. In this study, the avoidance behavior of the small native species (tits, chaffinch, sparrow and robin), staying next to the feeders while parakeets were feeding (what we referred to as PICE) may reduce the energetic cost of agonistic interactions but may have consequences for individuals. Changes in daily feeding routines may, for example, decrease the energetic gain of food for species with digestive constraints and force individuals to forage during less favorable times of the day (Bednekoff and Houston 1994). The indirect effects of the "wait-and-see" strategy on individuals (e.g., physiological condition) need to be investigated.

In the gardens we studied, the species most highly impacted by interference competition with the ring-necked parakeet was the starling $S$. vulgaris. The starling showed the highest temporal niche overlap (Fig. 2) and involvement in the TICE and PICE with the parakeet. Our results suggest strong competition between these two species in winter for supplementary food resources. A superior competitiveness of the ring-necked parakeet over the starling has already been suggested for the selection of nest cavities in the Mediterranean area (Dodaro and Battisti 2014). The starling can exploit a wide range of food items (Feare 1984) but during the winter, especially during long periods of frost when the availability of seeds and invertebrates is lower, the dependence of the starling on anthropogenic food resources is greater (Crick et al. 2002). Supplementary food at the breeding stage can increase hatching success, chick weight and the number of young fledged per starling pair (Crossner 1977). Considering that the populations of starlings have declined in Europe over the last three decades (Robinson et al. 2005), competition for supplementary food, especially in winter, could be a conservation issue for this species. The European robin was the earliest species at the bird feeders. This species showed the lowest temporal niche overlap (Fig. 2) and involvement in interference competition with the parakeet. These results support the hypothesis that differences in timing of foraging activity induce lower interspecific competition (Pianka 1973).

Most of the bird species present at the feeders in this study were "urban exploiters", species adapted to anthropogenic habitats depending almost entirely on humanprovided resources (Blair 2001; McKinney 2002), i.e., house sparrows and starlings; and "urban adapters"-great tits, blue tits, common chaffinch, Eurasian collared doves and European robins. Among all the species recorded in the video sequences, the ring-necked parakeet was the most abundant at the feeders. This was expected because the use of bird feeders represented half of its feeding time (Clergeau et al. 2009). A recent paper showed that supplementary food could favor invasive bird species in New Zealand and lead to an increase in their populations (Galbraith et al. 2015). Bird feeding is a very common activity and its effects on avian communities are controversial (for a review see Chace and Walsh 2006). In the UK, 7.4 million households provide supplementary food in bird feeders (Davies et al. 2009). These food sources may have positive effects on birds by increasing their clutch size (Brommer et al. 2004) and reducing their foraging time during the breeding season (Bollinger et al. 1990), which may lead to a higher recruitment. Negative effects of bird feeders have also been suggested, such as the increase of disease transmission or the failure of supplementary food to fully provide all the necessary nutrients for a bird (Robb et al. 2008). The impact of the ring-necked parakeet's presence at supplementary food sources on the integrity of the native species populations needs to be investigated. The number of ring-necked parakeets in our study area has increased exponentially over the last 20 years (Clergeau et al. 2015). 
Despite the fact that the number of parakeets has no effect on the frequency of PICE and TICE, the amount of food per individual may decrease with an increase in these visitors. The ring-necked parakeet being a gregarious and philopatric species, we might expect stronger competition between this species and the native species over the next few years. Aggressive behavior towards native species has already been reported for another invasive parakeet, the monk parakeet Myiopsitta monachus (Freeland 1973) and for other invasive birds such as the red-whiskered bulbul Pycnonotus jocosus and the red-vented bulbul Pycnonotus cafer (Jones 1996; Thibault et al. 2002). The roles of personality traits in invasion success need to be taken more fully into account for the impact assessment of invasive birds.

Acknowledgments We are grateful to the participants of this study, who kindly allowed access to their gardens. We thank Michael Paul, who helped improve an earlier version of this manuscript. We were financially supported by the Essonne local authority (Conseil Général). The authors would like to thank ParrotNet COST Action for constructive discussions.

\section{References}

Bednekoff PA, Houston AI (1994) Avian daily foraging patterns: effects of digestive constraints and variability. Evol Ecol $8: 36-52$

Blackburn TM, Cassey P, Lockwood JL (2009) The role of species traits in the establishment success of exotic birds. Glob Change Biol 15:2852-2860

Blair RB (2001) Birds and butterflies along urban gradients in two ecoregions of the US. In: Lockwood JL, McKinney ML (eds) Biotic homogenization. Springer, New York, pp 33-56

Bollinger PB, Bollinger EK, Malecki RA (1990) Tests of three hypotheses of hatching asynchrony in the common tern. Auk 107:696-706

Brommer JE, Karell P, Pietiäinen H (2004) Supplementary fed Ural owls increase their reproductive output with a one year time lag. Oecologia 139:354-358

Butler CJ (2003) Population biology of the introduced rose-ringed parakeet Psittacula krameri in the UK. Thesis, University of Oxford

Camargo JA (1993) Must dominance increase with the number of subordinate species in competitive interactions? J Theor Biol 161:537-542

Case TJ, Bolger D, Petren K (1994) Invasions and competitive displacement among house geckos in the tropical Pacific. Ecology 75(2):464-477

Cassey P, Blackburn TM, Sol D, Duncan RP, Lockwood JL (2004) Global pattern of introduction effort and establishment success in birds. Biol Lett 271(Suppl 6):S405-S408

Castro-Arellano I, Lacher TE Jr, Willig MR, Rangel TF (2010) Assessment of assemblage-wide temporal niche segregation using null models. Methods Ecol Evol 1:311-318

Chace JF, Walsh JJ (2006) Urban effects on native avifauna: a review. Landsc Urban Plan 74(1):46-69

Chapple DG, Simmonds SM, Wong BBM (2012) Can behavioral and personality traits influence the success of unintentional species introductions? Trends Ecol Evol 27(1):57-64
Clergeau P, Vergnes A (2011) Bird feeders may sustain feral roseringed parakeet Psittacula krameri in temperate Europe. Wildl Biol 17:248-252

Clergeau P, Vergnes A, Delanoue R (2009) La perruche à collier Psittacula krameri introduite en Ile-de-France: distribution et régime alimentaire. Alauda 77:121-132

Clergeau P, Leroy O, Lenancker P (2015) Dynamique de population de la perruche à collier Psittacula krameri introduite en Ile-deFrance. Alauda 83(3):165-174

Crick HQP, Robinson RA, Appleton GF, Clark NA, Rickard AD (2002) Investigation into the causes of the decline of starlings and house sparrows in Great Britain. BTO research report no. 290. DEFRA, Bristol

Crossner KA (1977) Natural selection and clutch size in the European starling. Ecology 58:885-892

Davies ZG, Fuller RA, Loram A, Irvine KN, Sims V, Gaston K (2009) A national scale inventory of resource provision for biodiversity within domestic gardens. Biol Conserv 142:761-771

Dodaro G, Battisti C (2014) Rose-ringed parakeet (Psittacula krameri) and starling (Sturnus vulgaris) syntopics in a Mediterranean urban park: evidence for competition in nest-site selection? Belg J Zool 144(1):5-14

Dunning JB (2007) CRC Handbook of avian body masses, 2nd edn. CRC, Boca Raton

Feare CJ (1984) The starling. Oxford University Press, New York

Feinsinger P, Spears EE, Poole RW (1981) A simple measure of niche breadth. Ecology 62:27-32

Freeland DB (1973) Some food preferences and aggressive behavior by monk parakeets. Wilson Bull 85(3):332-334

Galbraith JA, Beggs JR, Jones DN, Stanley MC (2015) Supplementary feeding restructures urban bird communities. PNAS 112(20):E2648-E2657

Grangier J, Lester PJ (2011) A novel interference behavior: invasive wasps remove ants from resources and drop them from a height. Biol Lett 7(5):664-667

Hernández-Brito D, Carrete M, Popa-Lisseanu AG, Ibáñez C, Tella JL (2014a) Crowding in the city: losing and winning competitors of an invasive bird. PLoS One 9(6):e100593

Hernández-Brito D, Luna A, Carrete M, Tella J (2014b) Alien roseringed parakeet (Psittacula krameri) attack black rats (Rattus rattus) sometimes resulting in death. Hystrix 25(2):121-123

Huenneke LF, Thomson JK (2003) Potential interference between a threatened endemic thistle and an invasive nonnative plant. Conserv Biol 9(2):416-425

Human KG, Gordon DM (1996) Exploitation and interference competition between the invasive Argentine ant, Linepithema humile, and native ant species. Oecologia 105:405-412

Johnson RN (1972) Aggression in man and animal. Saunders, Philadelphia

Jones CG (1996) Bird introductions to Mauritius: status and relationship with native birds. In: Holmes JS, Simons JR (eds) The introduction and naturalisation of birds. Stationery Office Publication Centre, London, pp 113-123

Lach L (2005) Interference and exploitation competition of three nectar-thieving invasive ant species. Insect Soc 52:257-262

Maurer BA (1984) Interference and exploitation in bird communities. Wilson Bull 96(3):380-395

McKinney ML (2002) Urbanization, biodiversity and conservation. Bioscience 52(10):883-890

McNamara JM, Houston AI, Lima SL (1994) Foraging routines of small birds in winter: a theoretical investigation. J Avian Biol 25:287-302

Menchetti M, Scalera R, Mori E (2014) First record of a possibly overlooked impact by alien parrot on a bat (Nyctalus leisleri). Hystrix 25(1):61-62 
Peck HL, Pringle HE, Marshall HH, Owens IPF, Lord AM (2014) Experimental evidence of impacts of an invasive parakeet on foraging behavior of native birds. Behav Ecol 25(3):582-590

Pianka ER (1973) The structure of lizard communities. Annu Rev Ecol Evol S 4:53-74

R Core Team (2015) R: a language and environment for statistical computing. R Foundation for Statistical Com puting, Vienna. Version 3.1.3. http://www.R-project.org/. Accessed 15 Jan 2015

Roback PJ, Askins RA (2005) Judicious use of multiple hypothesis tests. Conserv Biol 19:261-267

Robb GN, MacDonald RA, Chamberlain DE, Bearhop S (2008) Food for thought: supplementary feeding as a driver of ecological change in avian populations. Front Ecol Environ 6(9):476-484

Robinson RA, Siriwardena GM, Crick HQP (2005) Status and population trends of starling Sturnus vulgaris in Great Britain. Bird Study 52:252-260

Schoener TW (1983) Field experiments on interspecific competition. Am Nat 122(2):240-285

Siegel S (1956) Nonparametric statistics for behavioral sciences. McGraw-Hill, New York
Sol D, Timmermans S, Lefebvre L (2002) Behavioral flexibility and invasion success in birds. Anim Behav 63:495-502

Sol D, Maspons J, Vall-llosera M, Bartomeus I, García-Peña GE, Piñol J, Freckleton RP (2012) Unraveling the life history of successful invaders. Science 337:580-583

Strubbe D, Matthysen E (2009) Experimental evidence for nest-site competition between invasive ring-necked parakeets (Psittacula krameri) and native nuthatches (Sitta europaea). Biol Cons 142(8):1588-1594

Strubbe D, Matthysen E, Graham CH (2010) Assessing the potential impact of invasive ring-necked parakeets Psittacula krameri on native nuthatches Sitta europaea in Belgium. J Appl Ecol 47:549-557

Thibault JC, Martin JL, Penloup A, Meyer JY (2002) Understanding the decline and extinction of monarchs (Aves) in Polynesian Islands. Biol Conserv 108:161-174

Wilson E (1975) Sociobiology: the new synthesis. Harvard University Press, Cambridge 